\title{
Measuring the Quality of Pudendal Nerve Perineural Injections
}

Stanley Antolak, Jr, MD¹, Christopher Antolak, MD², and Lisa Lendway, PhD³

From: ${ }^{1}$ Center for Urologic and Pelvic Pain; St. Paul, $\mathrm{MN} ;{ }^{2}$ North Memorial

Medical Center; Silver Lake Clinic, St. Anthony, $\mathrm{MN}$; and ${ }^{3}$ Allina Health;

Minneapolis, MN

Address Correspondence: Stanley J. Antolak 838 138th St.

Amery, WI 54001

E-mail: sjantolak@aol.com

Disclaimer: There was no external funding in the preparation of this manuscript.

Conflict of interest: Each author certifies that he or she, or a member of his or her immediate family, has no commercial association

(i.e., consultancies,

stock ownership, equity interest, patent/licensing arrangements, etc.) that might pose a conflict of interest in connection with the submitted manuscript.

Manuscript received o1-14-2015

Revised manuscript

received: 10-29-2015 Accepted for publication: 11-10-2015

Free full manuscript: www.painphysicianjournal.

com
Background: Pudendal neuropathy is a tunnel syndrome characterized by pelvic pain and may include bowel, bladder, or sexual dysfunction or a combination of these. One treatment method, pudendal nerve perineural injections (PNPIs), uses infiltration of bupivacaine and corticosteroid around the nerve to provide symptom relief. Bupivacaine also anesthetizes the skin in the receptive field of the nerve that is injected. Bupivacaine offers rapid pain relief for several hours while corticosteroid provides delayed pain control often lasting 3 to 5 weeks. Not all pudendal nerve blocks may provide complete pain relief but long-term pain control from the steroid appears to be associated with immediate response to bupivacaine. We offer a method of evaluating the quality of a pudendal block on the day it is performed using pinprick sensation evaluation.

Objective: To demonstrate that pinprick sensory changes provide a simple and rapid method of measuring response to local anesthetic and pain reduction provided by a PNPI on the day it is performed. This response defines the quality of each PNPI.

Study Design: This is a case series based on retrospective review of a private practice database that is maintained by HealthEast hospitals in Minnesota. Database information includes standard physical examination, recording techniques, and treatment processes that had been in place for several years.

Setting: Private practice in United States.

Methods: Patients with a diagnosis of pudendal neuropathy are treated with PNPIs. Two hours after each block, 2 endpoints are measured: response to a sensory pinprick examination of the pudendal territory and difference in patient-reported pain level before and after nerve block. Fifty-three men from a private practice treating only pelvic pain received the treatment in 2005 . Reported pain level was not recorded for 2 patients.

Results: Bupivacaine in perineural injections produces varying degrees of analgesia or hypalgesia to pinprick. Normal pinprick response suggests pudendal nerves were not penetrated by bupivacaine. Patient responses varied from complete, i.e. all 6 branches anesthetized to none. Most men had 2 5 nerve branches anesthetized. One man had a single nerve branch that responded to bupivacaine. Three men failed to respond to local anesthetic.

Changes in pre-PNPI to post-PNPI pain scores were significantly decreased $(n=51, P$-value $<0.0001)$, indicating that bupivacaine in the PNPI reduced pain. Forty-one patients $(80.4 \%)$ indicated less pain after the procedure and only 2 patients $(4.0 \%)$ indicated more pain. The number of nerve branches successfully anesthetized was also significantly correlated with change in score. On average, an additional successful nerve branch anesthetized corresponded to a drop of about 0.66 in the change score $(n=51, P$ - value $=0.0005)$.

Conclusion: PNPIs relieve pain. Anesthesia affected all 6 pudendal nerve branches in only $13.2 \%$ of patients. Complete pain relief occurred in $39.2 \%$. This argues against use of perineural pudendal blockade as a diagnostic test. Pain relief after PNPI is associated with number of nerve branches that are anesthetized. At 2 hours after a PNPI its quality (the number of the 6 nerve branches with reduced response to pinprick from the perineural local anesthetic) is associated with subjective reduction of pain.

Key words: Pudendal neuralgia, chronic perineal pain, pudendal nerve block, sensory examination, neurologic examination, pain management, chronic pelvic pain syndrome

Pain Physician 2016; 19:299-306 
P udendal neuropathy is a tunnel syndrome characterized by pelvic pain and may include bowel, bladder, and sexual dysfunction (1). Treatment includes prevention of nerve damage, perineural steroid injections, and decompression surgery. The injection process, called pudendal nerve perineural injections (PNPIs) uses infiltration of bupivacaine and corticosteroid around the nerve. An effective pudendal block should anesthetize its 3 branches; the dorsal nerve of the penis (clitoris), the perineal nerve, and the inferior rectal nerve. Bupivacaine will anesthetize the skin and offers rapid pain relief for several hours. The corticosteroid provides delayed pain control often lasting 3 to 5 weeks. Soon after a bilateral block, the 6 branches can be evaluated individually for anesthetic response by comparing pinprick sensation between the pudendal sites and a normal site on the thigh. The number of branches anesthetized may vary from none to all 6 . Our observation is that the immediate pain control from bupivacaine and the long-term pain control from the steroid effect correlate with the immediate sensory response to the bupivacaine. Only the immediate skin anesthesia is considered a quality measure of the individual PNPI.

Three PNPIs, given at 4 week intervals, can often relieve neuropathic pelvic pain (2). The degree of pain relief varies in published reports (3). Not all pudendal nerve blocks provide complete pain relief. Even perfect quality PNPIs, with all 6 pudendal nerve branches demonstrating analgesia to pinprick, may provide only partial or possibly no pain relief.

The pudendal nerve is a mixed nerve commonly including fibers from spinal cord levels 5 2-3-4. Damage can occur from several causes such as sitting, exercises, falls, and childbirth. Such damage can result in pelvic pain with or without concurrent bowel, bladder, or sexual dysfunction. Pudendal neuropathy is a common problem and is usually a bilateral process (4). It is a tunnel syndrome analogous to the carpal tunnel syndrome

Table 1. Technique of measuring pudendal nerve branches: The medial thigh is used as a "normal" sensation site.

\begin{tabular}{|l|l||}
\hline Branch & $\begin{array}{l}\text { Placement of safety pin } \\
\text { (bilateral) }\end{array}$ \\
\hline $\begin{array}{l}\text { Dorsal nerve of penis } \\
\text { (clitoris) }\end{array}$ & Lateral aspect of glans (clitoris) \\
\hline Perineal nerve & Posterior scrotum (labium) \\
\hline Inferior rectal nerve & $\begin{array}{l}\text { Posterior anal mucocutaneous } \\
\text { junction }\end{array}$ \\
\hline
\end{tabular}

(5). The main nerve trunk includes all 3 branches as it passes between the sacrospinous and sacrotuberous ligaments. Damage occurs primarily in the space known as the interligamentary space between the sacrospinous and sacrotuberous ligaments. The site is also called the lobster claw or clamp. Nerve damage can affect one or more branches or only portions of the branches. This results in a broad array of confounding complaints. The secondary site of damage or nerve compression is in the pudendal (Alcock) canal.

As a tunnel syndrome, pudendal neuropathy can respond to treatments similar to the carpal tunnel syndrome. Treatments are sequential and include nerve protection and medications, perineural blockades using bupivacaine and steroids, and decompression surgery. Diagnosis of pudendal neuropathy is suspected from the symptom history, typically perineal pain that is aggravated by sitting or driving and reduced when sitting on a toilet seat. The problem is common but usually misdiagnosed as prostatitis, orchalgia, endometriosis, or vulvodynia. Any practitioner can make the diagnosis by finding abnormal pinprick sensation at one or more of the 3 pudendal nerve branches bilaterally (Table 1). Objective confirmation of neuropathy uses a pudendal nerve terminal motor latency test (PNTMLT) and a warm temperature threshold detection test that are easily performed by any physician. The warm temperature threshold detection test is a quantitative sensory test that measures the initial perception of warmth when a thermoprobe is warmed from a neutral temperature. It tests unmyelinated C-fibers. It is topical and not invasive (6). The PNTMLT tests large, myelinated fibers and is performed via digital rectal examination using a St. Mark's electrode to stimulate the pudendal nerve in its pathway medial to the ischial spine and receive the response at the rectal sphincter (7). Other available tests include somatosensory evoked potential testing and pelvic floor electromyography (8).

Bensignor et al (9), in Nantes, France, demonstrated that a series of 3 pudendal nerve perineural injections at 4 week intervals could consistently provide pain relief. Most sufferers require bilateral injections. In our experience, a single bilateral injection rarely cures a patient. The benefit of injections is cumulative and may peak as long as 6 weeks after completing a series of 3 PNPIs. The longest cure at our facilities following a series of PNPIs is 13 years. The results are measured using validated symptom scores, including the National Institutes of Health Chronic Pelvic Symptom Index and the American Urological Association Symptom Index 
$(10,11)$. Patients who fail to respond to PNPI are candidates for pudendal nerve decompression surgery.

\section{Methods}

In 2005 a group of 53 men with pudendal neuropathy underwent treatment using PNPI. Each was diagnosed by typical symptomatology and pinprick evaluation in the pudendal territory. Pudendal neuropathy was confirmed using 2 neurophysiologic tests: a warm detection threshold test and a pudendal nerve terminal motor latency test. All patients were treated with a nerve protection program and medications, typically gabapentin and amitriptyline. Nerve protection, a selfcare program, includes restricting sitting and restricting hip flexion exercises for example cycling, elliptical, step aerobics, jogging, abdomen crunches, and weight lifting. The men received 3 PNPIs. The anesthetic response to the first PNPI in the series is reported. Therapeutic response to corticosteroid response occurs in 3 to 5 weeks and is cumulative. It is not reported in this paper.

The first and second PNPIs use fluoroscopy and are given medial to the ischial spine between the sacrotuberous and sacrospinous ligaments. This interligamentary space is the major anatomical compression site identified during decompression surgery. Bupivacaine $0.25 \%, 6 \mathrm{~mL}$ and methylprednisolone $40 \mathrm{mg}$ are infiltrated around each nerve.

For fluoroscopically guided PNPI, the patient is placed prone on a fluoroscopy table. A pillow is placed under the ipsilateral hip, raising the pelvis approximately 30 degrees. This allows consistent vision of the ischial spine. The fluoroscope remains perpendicular to the table. The nerve pathway is usually immediately medial to the tip of the ischial spine. The tip of the ischial spine is marked on the skin of the buttock. After antiseptic preparation, a 22ga $12 \mathrm{~cm}$ needle is passed perpendicularly through the gluteus muscle until the ischial spine is gently touched. Then the needle is retracted several millimeters and is angled about $1-2$ $\mathrm{mm}$ medial to the tip of the bone (Fig. $1 \mathrm{~A}, \mathrm{~B}$, and C). It is advanced to the sacrotuberous ligament which is identified by palpable resistance to needle passage. This is usually 3 to $5 \mathrm{~mm}$ shallower than the bony ischial spine. Slow penetration of the sacrotuberous ligament is made into the interligamentary space. At this point, the needle stimulates paresthesias or pains in the pudendal territory. The needle is withdrawn $1-2 \mathrm{~mm}$ and medications are infiltrated into the interligamentary space.

The third PNPI requires a computed tomography (CT) scan to guide the needle into the narrow space of the pudendal canal. It is performed by an interventional radiologist and is described by Hough et al (12).

Because the pudendal nerve is generally completely formed into a single main trunk at the level of the ischial spine, a bilateral block into the interligamentary space should affect all 6 branches, 3 on each side.

Quality of anesthetic response to each block was

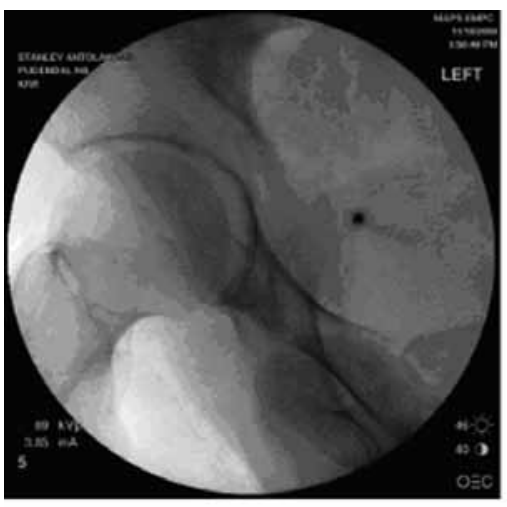

A

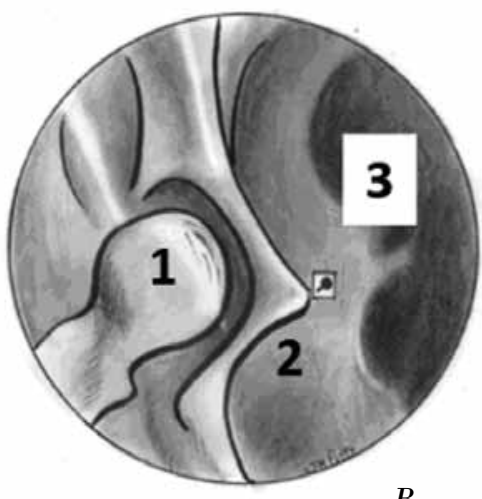

$B$

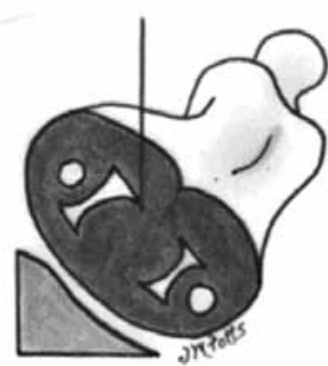

C

Illustrations provided by Dr. Jeanette Potts

Fig. 1. A, Fluoroscopic guided PNPI. Hub of needle medial to ischial spine. B, 1. Femoral head. 2. Ischial spine with hub of needle medially 1-2 mm. 3. Greater sciatic notch. C, Position for PNPI. Ipsilateral hip elevated. Needle is inserted perpendicular to floor. 
evaluated 2 hours after the PNPI using a safety pin. The time interval permits adequate time for anesthesia to penetrate the nerve. Pinprick examination is performed at each of 6 pudendal nerve branches. The dorsal nerve of the penis is tested at the mid-glans laterally to avoid overlap at the dorsum. The perineal nerve is tested at the reflection of the scrotum from the perineum anterior to the rectum. The inferior rectal nerve territory is examined posterior to the coronal midline of the anus which is the separation of the 2 pudendal sensory branches to the perineum. Men stand for the testing. Women, not included in this study, lie supine in a "frog leg" position. A light pinprick at the inner thigh is made to remind of the normal pinprick sensation. Then the patient is asked to describe the intensity of pinprick at each pudendal site. Analgesia, with no sensation of pain or touch, is the ideal response to the PNPI. Hypalgesia is a partial anesthetic response with some degree of reduction of the pinprick sensation. It is a positive response but indicates a less thorough absorption of the bupivacaine. Normal sensation or hyperalgesia indicate no nerve absorption of the anesthetic.

Quality can also be measured by patient subjective description of pain level preceding PNPI and prior to the 2 hour post block examination. Patients rate pain from 0 to 10 , with 0 being no pain and 10 a pain level that would require intravenous narcotics. This rating is performed by a nurse immediately before the injection pain. Subsequently the examining physician asks about the pain level at 2 hours after the PNPI which is before the pinprick sensation is tested.

\section{Results}

Fifty-three patients underwent pinprick sensory testing after PNPI. Their pain levels were also recorded before and after the PNPI. Two patients had missing pain levels. Forty-nine men had one to 6 nerve branches (sites) responding to pinprick with analgesia or hypalgesia. The proportion with pain level of 0/10 at the 2 hour exam was greater for those with 5 or 6 sites responding $(75 \%)$ than those with less sites responding (Table 2). Four of the patients in the cohort $(8.1 \%)$ demonstrated no anesthetic response following PNPI and none of these patients noted pain reduction at the 2 hour follow-up examination. Pain was completely relieved at 2 hours after the first PNPI in 20 men (39.2\%).

Only 6 men had a perfect PNPI, where all 6 nerve branches were anesthetized. One of these men had no pain relief. Four men had no sensory response to the bupivacaine; there was no change in their pre- and post-pain scores. The remaining men had one to 5 branches responding with analgesia or hypalgesia. Of these, 2 men had increased pain at 2 hours after PNPI.

A paired t-test showed a significant decrease in pain score $(n=51, P$-value $<0.0001)$ after PNPI. Fig. 2 shows a plot of the change score, as measured by post-PNPI pain score minus pre-PNPI pain score, versus number of nerve branches anesthetized, achieving either hypalgesia or analgesia. Simple linear regression confirmed that there is a significant relationship between change score and number of nerve branches anesthetized $(P$ value $=0.0005$ ). Specifically, patients with an additional site anesthetized have changed scores that are 0.66 lower, on average.

\section{Discussion}

Sensory examination with a pinprick is commonly recognized as being adequate for detection and characterization of gross abnormality in a dermatome/ receptive field. Zuelzer (13), in 1915, described pinprick sensory changes that demonstrated pudendal neuropathy in women with sterile voiding symptom such as interstitial cystitis. Turner and Marinoff (14), in 1991, advocated pinprick sensory testing in women with vulvodynia in order to distinguish the subset of their patients who had pudendal neuropathy. The testing is simple, rapid, and inexpensive, and can be performed by any alert and interested practitioner.

PNPIs were used by French pioneers in the treatment of pudendal neuropathy. They noted mixed results after a series of multiple PNPIs. Bensignor et al (9) described therapeutic benefit, from fair pain reduction to cure of pain, in $69 \%$ of patients at $12-78$ months after one to 4 PNPIs (average 2.1). Amarenco et al (15) noted $57 \%$ immediate response but only $15 \%$ excellent or good long-term results at 12 months after the

Table 2. Responses following a single transgluteal pudendal PNPI ( $n=51)$.

\begin{tabular}{|l|c|c|c|c|}
\hline Number of sites responding & 5 or 6 & 3 or 4 & 1 or 2 & 0 \\
\hline Number of patients & 16 & 20 & 11 & 4 \\
\hline Pain level 0/10 at 2 hour exam & $12(75 \%)$ & $5(25 \%)$ & $3(27.3 \%)$ & $0(0 \%)$ \\
\hline
\end{tabular}




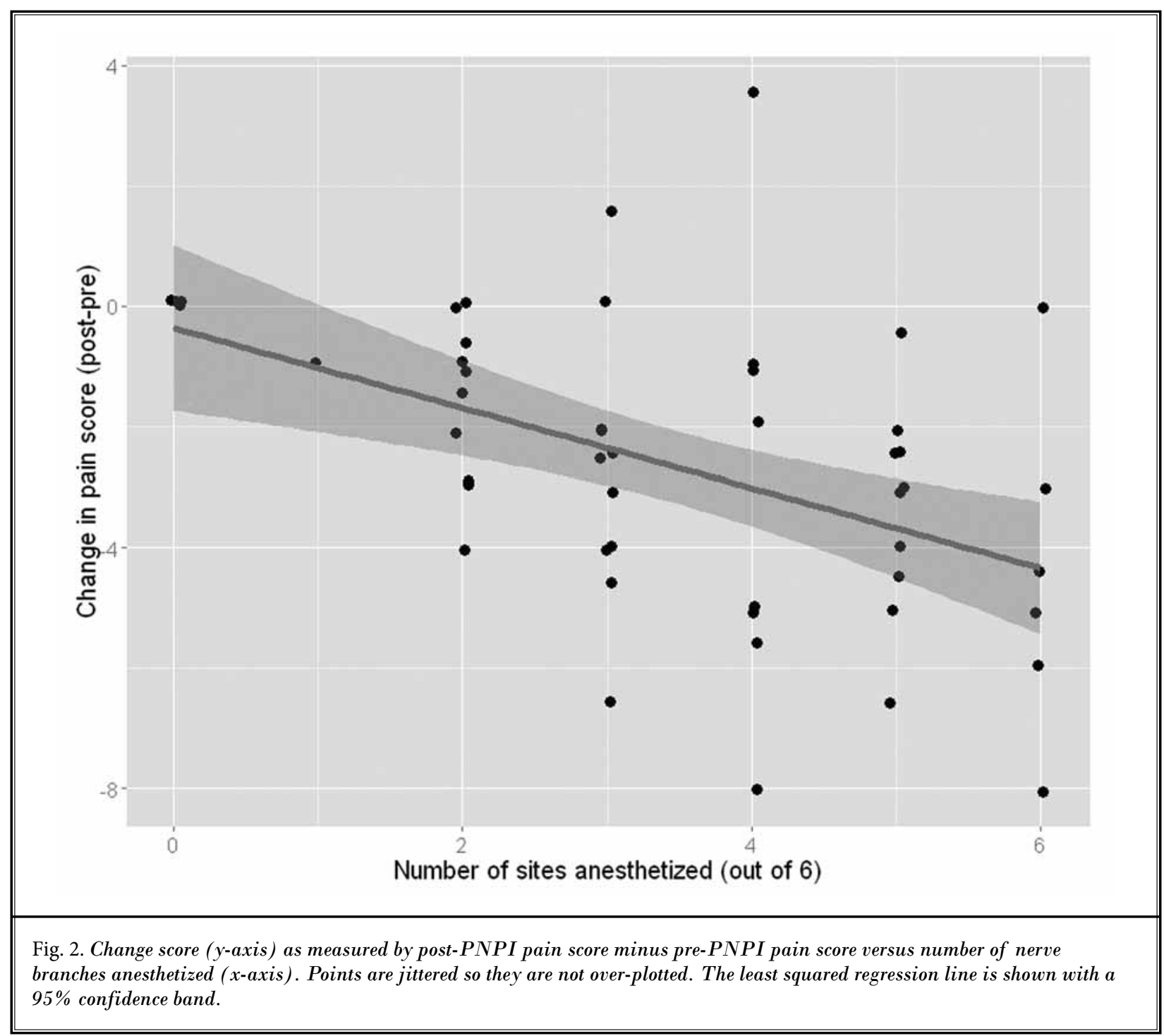

last PNPI. Robert et al (16) indicated $65 \%-70 \%$ cured by $\mathrm{x}$-ray or CT-guided PNPI. Comparisons are difficult because these authors did not always use the same technique. Some gave only a single PNPI; others gave multiple PNPIs but not at consistent intervals. Injection sites were not consistent.

None of these authors monitored quality on the day of the PNPI. They report results of pudendal nerve blocks differently. Thoumas et al (3) describe anesthesia "in the cutaneous territory of the pudendal nerve" in over $90 \%$ of patients. They do not indicate the postblock interval to examination or if the territory of each specific nerve branch was tested (3). Amarenco et al (8) report that infiltrations at the ischial spine were success- ful in $57 \%$ but give no further information. Recently, Vancaille et al (17) identified numbness to touch with cotton at 2 hours after transvaginal pudendal block in 61 of 66 women (92.4\%). They did not report the sites examined or the number of pudendal branches that responded to PNPI. In their series, reduction in one or more pain symptoms occurred in about $86.9 \%$.

Bellingham et al (18) discuss the quality of PNPI in a comparison of 2 methods of PNPI. They used ultrasound and fluoroscopic guidance on opposite sides in each patient. Pinprick and cold temperature sensation were measured in "all areas of the perineum." This test region might have examined both the perineal branch and the perineal distribution of the inferior rectal nerve. 
They did not evaluate the dorsal nerve of the clitoris (penis) and may not have had "complete" responses. Nine of the 23 patients did not have anesthesia to pinprick (19.6\%). In our experience a 20 minute interval is too short for complete response to the bupivacaine. Some of our patients had subjective pain that was still decreasing at the 2 hour office follow-up. Conversely occasionally patients note short-term pain control with pain rapidly returning prior to the 2 hour follow-up. It is possible that Bellingham et al's (18) results may have differed if examination had been performed after a longer time interval.

The present paper attempts to be more precise in measuring quality by testing pinprick in all 6 branches of the pudendal nerve. The perineal innervation is rather precisely divided between the perineal nerve and the inferior rectal nerve at the coronal midline of the anus. None of the previous quality papers separated perineal sensory testing, specifically Bellingham et al (18) who tested "all areas of the perineum."

In the present series 49 of 53 patients had one or more of the 6 pudendal nerve branches anesthetized resulting in hypalgesia or analgesia. This could be calculated as a $92.5 \%$ success rate if we were reporting responses in the pudendal territory or "in all areas of the perineum." Instead, measuring the anesthetic response precisely in each of 6 pudendal nerve branches, hypalgesia or complete analgesia occurred in 183 of $318(57.5 \%)$ nerve branches in which response should have occurred had the blocks been consistently "perfect." Using the end point of pain reduction following a PNPI, 41 of $51(80.4 \%)$ had pain reduction after this single PNPI that was the first in a planned series of 3 PNPIs. Post-PNPI pain levels were not recorded in 2 men.

Achievement of good quality PNPI is difficult and perfect results are not obtained after every attempt. Several factors may cause inadequate results including:

1. Good pudendal paresthesias during placement of PNPI needle with poor pinprick response.

a. This might indicate that the needle is not adjacent to the nerve during injection of medications. Surgical findings often demonstrate multiple, complex layers of perineural fascias and ligamentous fibers that would not permit precise placement or diffusion of medications.

b. The injection needle may depress the sacrotuberous ligament, compressing the nerve anteriorly against the sacrospinous ligament, resulting in paresthesias but without penetrating into the interligamentary space. c. Resistance to local anesthetics is reported (19). The men in this series had no history of failure of dental injections.

2. The patient does not understand what needle paresthesias to describe.

a. We explain as the needle traverses the gluteus muscle, touches the ischial spine, and penetrates the sacrotuberous ligament which is typically painful.

b. We explain to the patient to expect tingling, pressure, or pain at the rectum, scrotum, perineum, or penis.

c. If the patient complains of such "signals" or pain, we ask if the pain is deep, at the needle tip, or is in one of the expected pudendal branches.

3. When pain reduction does not occur despite analgesia at all 6 sites, there may be a compression proximal to the PNPI. This is uncommon.

a. It is seen most often following the distal, CT guided PNPI into the Alcock canal and indicates compression at the clamp in the interligamentous space.

b. In one patient not in this cohort, pinprick anesthesia without pain relief followed the PNPI at the ischial spine. Magnetic resonance imaging (MRI) of the sacral spine identified a sacral cord tumor.

4. More frequent causes of persistent pain following adequate PNPI are peripheral neuropathies at the perimeter of the pudendal nerve receptive field. Two frequent causes are ilioinguinal and iliohypogastric neuropathies affecting inguinal or scrotal pain and the Maigne syndrome (thoracolumbar junction syndrome) affecting suprapubic and inguinal pain. Middle cluneal neuropathy and abdominal cutaneous neuropathies also occur. Symptoms in the perineal branch of the posterior femoral cutaneous nerve can be confusing. The nerve overlaps pudendal territory in the anal, perineal, and inguinal areas.

Paresthesias are expected during needle placement although significant pain is uncommon. Bensignor et al (9) did not seek paresthesias but did an anatomical placement medial to the tip of the ischial spine into the interligamentary space. This space can be identified by needle passage through the sacrotuberous ligament and can be noticed by the resistance of that ligament during advancement of the needle. 
PNPI is a therapeutic tool and should not be used for diagnosis. We have always emphasized that pudendal neuropathy is confirmed by neurophysiologic testing. Labat et al (20) consider that a diagnostic block is necessary for the diagnosis of pudendal neuropathy. This point is especially relevant considering that in our series, the first PNPI gave total pain relief, that is a postPNPI pain level of 0 , in only 20 of 51 men. Thus, only $39.2 \%$ of our cohort would have a positive diagnosis of pudendal neuropathy by a "diagnostic block." In another study, complete pain relief occurred in only $57 \%$ of patients, meaning that $43 \%$ of the patients would not have been diagnosed with pudendal neuropathy based on a single diagnostic block (15). Bellingham et al (18) state that "a successful pudendal nerve block is crucial to the diagnosis of pudendal neuropathy." However 9 of 46 nerves injected had no anesthetic response. This would eliminate $19.4 \%$ of their patients from the diagnostic criterion. We strongly encourage the use of simple in-office neurophysiologic testing that will diagnose $100 \%$ of pudendal neuropathy patients (unpublished data). Pudendal blocks should be abandoned as a diagnostic test.

There are some limitations to this study. Only a single fluoroscopically guided pudendal nerve perineural injection is analyzed for its immediate effectiveness in a small cohort of the thousands of injections we have performed. We are defining the pinprick responses to bupivacaine as the measure of "quality" whereas other authors may prefer the pain reduction. We feel pain reduction is affected by other variables that cannot be measured such as severity of nerve compression. Quality of CT-guided blocks into the pudendal canal will be discussed in a future publication. Both men and women are injected using the same technique and we do not feel that there should be any gender variation when performing PNPI.

\section{Conclusion}

In conclusion, PNPIs can effectively control chronic pelvic pain caused by pudendal neuropathy on the day of injection (bupivacaine effect) and long-term (steroid effect). The quality is determined 2 hours after the PNPI by pinprick anesthesia in the 6 pudendal nerve branches. A second end point is the numerical pain reduction after the PNPI. Immediate pain control after a single PNPI as measured by the change in numerical score is affected by the quality of the block as measured by the number of nerve branches responding with hypalgesia or analgesia. PNPIs should not be used to diagnose pudendal neuropathy. A single PNPI results in complete pain control in approximately 40 to $85 \%$ of patients in published reports. In published articles, best responses to a single PNPI are $85 \%$ complete pain control. Lack of consistent, complete pain relief indicates that diagnosis of pudendal neuropathy should only be made using neurophysiologic tests. A series of 3 PNPIs can be therapeutic for many months $(1,3,4,8)$.

\section{Acknowledgments}

Ms. Claudia Greene reviewed and recorded the data used in this study under guidance of Ms. Susan Mehle. The Pudendal Neuralgia Foundation provided level 1 funding. There are no conflicts of interests nor financial disclosures regarding the authors.

\section{References}

1. Robert R, Labat JJ, Lehur PA, Glemain $\mathrm{P}$, Armstrong O, Le Borgne J, Barbin JY. Re'flexions cliniques, neurophyiologiques et therapeutiques a partir de donne'es anatomiques sur le nerf pudendal (honteux interne) lors de certaines algies pe'rine'ales. Chirurgie 1989; 115:515-520.

2. McDonald JS, Spigos DG. Computed tomography - guided pudendal block for treatment of pelvic pain due to pudendal neuropathy. Obstet Gynecol 2000; 95:306-309.

3. Thoumas D, Leroi AM, Mauillon J, Muller JM, Benozio M, Denis P, Freger P. Pudendal neuralgia: CT-guided pu- dendal nerve block technique. Abdom Imaging 1999; 24:309-312.

4. Antolak SJ. Interventions in managing male pelvic pain. In: Manchikanti L, Singh V (eds). Interventional Techniques in Chronic Non-Spinal Pain. ASIPP Publishing, Paducah KY, 2009, pp 303-318.

5. Pécina MM, Krmpotic-Nemanic J, Markiewitz AD. Pudendal nerve syndrome (syndrome of Alcock's tunnel). In: Pécina MM, Krmpotic-Nemanic J, Markiewitz AD (eds). Tunnel Syndromes: Peripheral Nerve Compression Syndromes. 3rd ed. CRC Press LLC, Boca Raton, 2001, pp 191-194.

6. Bleustein $\mathrm{CB}$, Eckholdt $\mathrm{H}$, Arezzo $\mathrm{JC}$,
Melman A. Quantitative somatosensory testing of the penis: Optimizing the clinical neurological examination. J Urol 2003; 169:2266-2269.

7. Tetzschner T, Sorensen M, Lose G, Christiansen J. Pudendal nerve recovery after a non-instrumented vaginal delivery. Int Urogynecol ] Pelvic Floor Dysfunct 1996; 7:102-104.

8. Amarenco G, Ismael SS, Bayle B, Denys $P$, Kerdraon J. Electrophysiological analysis of pudendal neuropathy following traction. Muscle Nerve 2001; 24:116-119.

9. Bensignor MF, Labat JJ, Robert R, Ducrot P. Diagnostic and therapeutic nerve blocks for patients with perineal non- 
malignant pain. Abstract, 8th World Congress on Pain 1996; p. 56.

10. Litwin MS, Mcnaughton-Collins $M$, Fowler FJ Jr, Nickel JC, Calhoun EA, Pontari MA, Alexander RB, Farrar JT, O'Leary MP. The National Institutes of Health chronic prostatitis symptom index: Development and validation of a new outcomes measure. J Urol 1999; 162:369-375.

11. Barry MJ, Fowler FJ Jr., O'Leary MP, Bruskewitz RC, Holtgrewe HL, Mebust WK, Cockett AT. The American Urological Association symptom index for benign prostatic hyperplasia. J Urol 1992; 148:1549-1557.

12. Hough, DM, Wittenberg KH, Pawlina W, Maus TP, King BF, Vrtiska TJ, Farrell MA, Antolak SJ Jr. Chronic perineal pain caused by pudendal nerve entrapment: Anatomy and CT-guided perineural injection technique. Am J Roentgenol 2003; 181:561-567.

13. Zuelzer G. Reizung des Nervus pudendus (Neuralgie). Berlin Klin Woch 1915; 49:1280-1281.

14. Turner MLC, Marinoff SC. Pudendal neuralgia. Am J Obstet Gynecol 1991; 165:1233-1236.

15. Amarenco G, Kerdraon J, Bouju P, Bu$\operatorname{det}$ C, LeCocquen A, Bosc S, Goldet R. Treatments of perineal neuralgia caused by involvement of the pudendal nerve. Rev Neurol 1997; 153:331-334 [in French].

16. Robert R, Prat-pradat D, Labat JJ, Bensignor M, Raoul S, Rebai R, Leborgne J. Anatomic basis of chronic perineal pain: Role of the pudendal nerve. Surg Radiol
Anat 1998; 20:93-98.

17. Vancaille T, Eggermont J, Armstrong G, Jarvis S, Liu J, Beg N. Response to pudendal nerve block in women with pudendal neuralgia. Pain Med 2012; 13:596-603.

18. Bellingham GA, Bhatia A, Chan CW, Peng PW. Randomized controlled trial comparing pudendal nerve block under ultrasound and fluoroscopic guidance. Reg Anesth Pain Med 2012; 37:262-266.

19. Trescot AM. Local anesthetic "resistance." Pain Physician 2003; 6:291-293.

20. Labat JJ, Riant T, Robert R, Amarenco G, Lefaucheur JP, Rigaud J. Diagnostic criteria for pudendal neuralgia by pudendal nerve entrapment (Nantes criteria). Neurourol Urodyn 2008; 27:306-310. 